\title{
$=$
}

\section{Escala para la valoración de las competencias de producción científica en docentes de Educación Superior}

\section{Scale for the assessment of scientific production skills in higher education teachers}

\author{
HERNÁNDEZ-SUÁREZ, César A. ${ }^{1}$ \\ PRADA-NÚÑEZ, Raúl ${ }^{2}$ \\ AVENDAÑO-CASTRO, William R. ${ }^{3}$
}

\begin{abstract}
Resumen
En la universidad todo profesor debe estar imbuido tanto en la actividad científica como en la docencia, y por ello resulta de interés conocer las actitudes de los docentes en torno a la producción de conocimiento científico. Se realizó una investigación cuantitativa, centrada en el análisis descriptivo correlacional-causal de los ítems de un test y un análisis factorial confirmatorio con 130 docentes. Como conclusión, el modelo generado resulta suficientemente explicativo de las variables relacionadas con la producción científica del conocimiento.

Palabras clave: competencias científicas, producción científica, docentes universitarios, análisis factorial.

Abstract

At university, every teacher must be imbued with both scientific activity and teaching, and it is therefore of interest to know the attitudes of teachers towards the production of scientific knowledge. A quantitative investigation was carried out, centred on the descriptive correlation-causal analysis of the items in a test and a confirmatory factor analysis with 130 teachers. In conclusion, the model generated is sufficiently explanatory of the variables related to the scientific production of knowledge.

key words: scientific skills, scientific production, university teachers, factorial analysis.
\end{abstract}

\section{Introducción}

La producción de conocimiento, junto con la docencia y la proyección social, conforman la base fundamental sobre la que se asientan las instituciones de educación superior (Sánchez, 2007; Bogado de Scheid y Fedoruk, 2011). En efecto, las universidades son por antonomasia los espacios del saber: en ellas se procura reunir, enseñar y divulgar cuanto es posible conocer, pero también constituyen uno de los principales núcleos en los que se genera conocimiento (Plastino, 2008). Este ha sido históricamente su propósito (Beraza y Rodríguez, 2007). Y lo sigue siendo (Sigal, 2009).

En Colombia, "el 70\% de la investigación que se hace en el país se gesta en el interior de las universidades" (Ministerio de Educación Nacional, 2005, p. 1). Las instituciones de educación superior en el contexto colombiano

\footnotetext{
${ }^{1}$ Magister en Educación. Docente investigador Facultad de Educación, Artes y Humanidades. Universidad Francisco de Paula Santander. Correo: cesaraugusto@ufps.edu.co

${ }^{2}$ Magister en ingeniería de análisis de datos, mejora de procesos y toma de decisiones. Docente investigador Facultad de Educación, Artes y Humanidades. Universidad Francisco de Paula Santander. Correo: raulprada@ufps.edu.co

${ }^{3}$ PhD en Ciencias Sociales y Humanas. Docente investigador. Universidad Francisco de Paula Santander. Correo: williamavendano@ufps.edu.co
} 
desempeñan un rol de suma importancia en la formación de recursos humanos y en la generación, desarrollo, transferencia de conocimiento y tecnologías que respondan a los requerimientos de la sociedad (Gamboa, Hernández y Prada, 2020). Además, la investigación se plasma como una prioridad para la universidad y es coherente con las exigencias del sistema de aseguramiento de la calidad en Colombia, sin embargo, estos discursos no se visibilizan ni permean la realidad institucional (Vergara, Gamboa y Hernández, 2018).

La producción de conocimiento, como es natural, se ha ido encauzando según el transcurso de los tiempos (Palacio, 2010). Y sin ser la única vía, la investigación científica es en la actualidad una de las tareas esenciales de las instituciones de educación superior y de su cuerpo docente (Ruiz, 2010). En efecto, la idea de que los investigadores y los docentes cumplen en la institución tareas especializadas y exclusivas ha cedido a una visión integradora de la labor del profesor universitario, que debe ejercer ambos roles a la vez (Mas, 2011). Cada vez hay menos profesores consagrados exclusivamente al estudio del saber acumulado para transmitirlo luego en sus clases. Y cada vez son más escasos los investigadores que se dedican únicamente a sus proyectos. El perfil del docente investigador ha triunfado en la universidad contemporánea por cuanto se entiende que para enseñar conviene también comprender los procesos de la ciencia y que toda investigación sin proyección sobre la formación de los futuros profesionales está al margen del carácter formativo de las generaciones de relevo, que han de serlo no solo en el ejercicio profesional, sino además en la productividad científica futura (Sancho, 2001). Por otra parte, distintos organismos internacionales, tales como la Organisation for Economic Co-operation and Develoment - OECD (2007), han insistido en que las posibilidades de desarrollo de las sociedades actuales están estrechamente vinculadas con el diseño y ejecución de políticas públicas que se ordenen en torno a oportunidades basadas en el conocimiento, tareas en las que la participación de las universidades y los universitarios resulta prioritaria.

A esta consideración holística del rol del profesor universitario se suma la evaluación externa (entre pares y organismos administrativos diversos) del prestigio y acreditación de los propios docentes y de las instituciones a las que están adscritos. En efecto, las evaluaciones sobre la calidad educativa y la productividad científica forman parte de la visión y práctica de la universidad contemporánea (Yoguez, 2009) y requieren que el personal docente esté altamente comprometido tanto con su actividad de aula como con la investigación y difusión científica (Orler, 2012). Dicho de otra manera, en la universidad actual todo profesor debe estar imbuido en la actividad científica en una medida semejante a como lo está con la docente.

En este sentido, resulta de interés máximo conocer las actitudes de los docentes universitarios en torno a la producción de conocimiento científico y su grado de compromiso con las actividades de investigación dentro de su institución, tanto por lo que concierne a su propia carrera académica como a su contribución a la proyección de la universidad a la que se haya adscrito en el plano investigativo.

El estudio de las actitudes ha resultado sumamente atractivo dentro de las ciencias sociales por cuanto permite conocer la manera en que se construyen, fundamentan y modifican la cognición y la afectividad sobre el mundo y las cosas y por su eventual valor predictivo de las conductas de las personas (Sabater, 1989; Blanco, 2001). En efecto, una actitud se define en general como una predisposición para actuar de un determinado modo ante una situación, evento, hecho o persona. Summers (1984), afirma que una actitud es la suma total de "inclinaciones y sentimientos humanos, prejuicios y distorsiones, nociones preconcebidas, temores y convicciones acerca de un asunto determinado" (p. 158). Y aunque no siempre existe una correspondencia estricta entre actitud y reacción, sí que es cierto que el conocimiento de las actitudes de las personas permite inferir en cierta medida sus comportamientos (Rodríguez, 2007).

El estudio de las actitudes, en efecto, constituye un recurso de gran valor para el conocimiento de las motivaciones y disposiciones que influyen sobre el comportamiento de las personas. Aunque carecen de carácter predictivo, informan certeramente acerca de las razones y convencimientos que predisponen a actuar de una 
determinada manera. De ahí su interés para las ciencias sociales, que más que en predecir las acciones de las personas, están interesadas en sus motivaciones (Canto, 1994).

En el caso concreto de las actitudes hacia la investigación científica entre docentes universitarios, un estudio de esta naturaleza tendría la utilidad de ayudar a comprender las concepciones que poseen los profesores sobre la importancia de la investigación para su trayectoria académica personal y la de su universidad, el modo en que evalúan sus propias capacidades y destrezas y el grado de compromiso que asumen frente a estas responsabilidades. Como se sabe, la producción científica abarca una serie de trabajos y procesos, que ven desde la ideación del proyecto (cuya génesis se encuentra en una observación del entorno con perspectiva científica y en el estudio acucioso de literatura especializada) hasta su ejecución (mediante la aplicación del método científico) y la divulgación de los resultados (en los foros y publicaciones adecuados). Cada una de estas etapas involucra habilidades y tareas múltiples. Puesto que la producción científica es multidimensional, conviene desglosar la actitud de los investigadores hacia cada una de los componentes, etapas y dimensiones de la investigación, lo mismo que la percepción que tiene sobre sus propias destrezas para la ejecución de cada uno. Este reconocimiento desglosado de las actitudes de los docentes investigadores permitiría una consideración de los problemas a los que se enfrentan en su trabajo productivo y orientarían el modo en el que las instituciones pueden contribuir con el fortalecimiento de los aspectos consolidados y la subsanación de debilidades y deficiencias.

En este sentido, este artículo informa sobre los resultados de un estudio cuyo propósito ha consistido en el diseño y validación de una escala para la medición de actitudes hacia la productividad científica entre docentes de educación superior en Colombia.

El estudio se circunscribe concretamente a la ciudad de Cúcuta y la muestra proviene del profesorado de la Universidad Francisco de Paula Santander - UFPS. Sin embargo, puesto que la pesquisa se ha concentrado en la validación del instrumento, la escala puede ser aplicada en otros contextos, nacionales e incluso iberoamericanos, a fin de obtener información más general sobre actitudes de los docentes universitarios en torno a la investigación.

\subsection{Hacer Ciencia: la producción científica de conocimiento}

La ciencia es un modo de conocimiento. Ante la complejidad del mundo y de las cosas (y aun la suya propia) el hombre ha procurado explicaciones diversas según distintos puntos de vista (filosófico, teológico, humanista, artístico, entre muchos otros). Uno de estos modos de aproximación a la realidad para procurar su comprensión es el de la ciencia, que consiste básicamente en la aplicación de un método controlado de observación de los fenómenos de la naturaleza (a veces con intervención sobre el objeto de estudio), a fin de descubrir, por medio de la inducción, las leyes que explican tales hechos.

El modo de conducirse para la producción del conocimiento científico es lo que se ha dado en llamar el método científico, que no es otra cosa que una serie ordenada de procedimientos validados por múltiples experiencias que permiten cierto grado de objetividad en la descripción de los fenómenos estudiados. Este método se activa mediante una pregunta de investigación acerca de un hecho o fenómeno, la elección del modo más adecuado para aproximarse al objeto de estudio, la aplicación de los protocolos elegidos, la obtención de datos y su posterior explicación con arreglo (si existe) a una determinada teoría.

Este método ha adoptado formas múltiples según el progreso de las ciencias y las disciplinas y de acuerdo con la naturaleza de los hechos estudiados. En cualquier caso, su validez ha sido probada en múltiples ocasiones y hoy constituye una de las fórmulas más apreciadas para la obtención de nuevos saberes. 
Desde de su creación, las universidades han sido (y siguen siendo) uno de los principales repositorios del saber; pero también de la generación de nuevos conocimientos. Y una de las vías para su obtención ha sido el de la ciencia. Universidad y saber, conocimiento y ciencia son, pues, entidades intrínsecamente ligadas. Quienes desde las universidades se dedican a la investigación deben, por otra parte, poseer destreza suficiente tanto para la producción de conocimiento con arreglo la tradición científica como para situar sus resultados en el panorama del saber acumulado y ofrecer la posibilidad de que se hagan públicos para beneficio social.

En efecto, además de la generación del conocimiento hay otros dos aspectos fundamentales ligados a la ciencia que explican su continuidad y el progreso en el conocimiento: la fundamentación del saber nuevo en el cúmulo de conocimientos previos y la difusión de los descubrimientos. En efecto, el conocimiento en general, y el científico en particular, son de carácter acumulativo: ningún saber surge de la nada, sino que se entronca en una tradición. Y por otra parte, el conocimiento científico sin divulgación pierde trascendencia y repercusión social.

Así las cosas, el trabajo del científico no solo tiene que ver con la generación de conocimiento, sino con la formación previa y constante en los asuntos de las ciencias y disciplina en las que incursiona; pero su labor también se extiende a la capacidad para hacer públicos sus hallazgos a fin de que repercutan en el progreso de las ciencias y en el bienestar social. En este sentido, todo científico debe desarrollar habilidades para la localización y aprovechamiento de la información científica a disposición para nutrir sus perspectivas en el planteamiento de problemas de investigación y su capacidad analítica a la hora de dar explicación a los hechos que describe. Tradicionalmente, estas labores se cumplieron mediante el estudio de libros de textos, manuales y artículos científicos, por la asistencia a eventos especializados y mediante el contacto y debate con los colegas. A estas maneras tradicionales de sustentar su visión teórica y su formación científica se suman ahora las posibilidades prácticamente infinitas que proporcionan los medios digitales. En este sentido, nuevas habilidades resultan ahora indispensables: por una parte, el desempeño en el manejo de las nuevas tecnologías y, por otra, la capacidad para ubicar, evaluar y elegir del universo de datos al alcance aquellos que sean verdaderamente relevantes según los propósitos de la investigación.

Asimismo, resulta indispensable una visión acerca de los escenarios actuales de la divulgación científica. Además de la asistencia a eventos especializados y de los sistemas editoriales tradicionales de difusión de conocimiento, se encuentran disponibles de un tiempo a esta parte una multiplicidad de medios electrónicos: revistas virtuales, foros, blogs, repositorios institucionales, páginas web, plataformas educativas interactivas, entre los más destacados. Y aquí, de nuevo, se exige del científico contemporáneo la capacidad de discernimiento sobre el escenario más apropiado para hacer de dominio público los conocimientos nuevos que su trabajo aporta. Y al igual que la selección del soporte, es de vital importancia el manejo de las estrategias discursivas propias de la tradición y de los formatos de divulgación de la ciencia, a los que hay que sumar los más recientes propios de los espacios digitales. En efecto, uno de los aspectos fundamentales para la publicación científica tiene que ver con el conocimiento y manejo adecuado de los estándares propios de la comunidad científica. Escribir un artículo científico (o cualquier otro formato equivalente) requiere de su autor el acomodamiento del informe a unas determinadas pautas editoriales, que son parte de la tradición científica y facilitan la continuidad y progreso del conocimiento.

En resumen, hacer ciencia consiste principalmente en generar conocimiento nuevo conforme al método científico, y estar en capacidad, además, de relacionarlo con los saberes disciplinares previos y poner a disposición de los pares y del público interesado, en los espacios adecuados, los resultados de la investigación.

\subsection{Actitudes hacia la investigación y la producción científica entre docentes universitarios}

En el caso de las actitudes hacia la producción científica, el objeto de la actitud es, como no puede ser de otro modo, asumir los procesos de génesis de nuevo conocimiento por aplicación de las metodologías propias de las 
distintas ciencias y disciplinas. En palabras de Blanco y Alvarado (2005), la actitud hacia la producción de investigación

es una predisposición organizada para pensar acerca del proceso de investigación científico que incluye el conjunto de creencias, valores y conocimientos que el docente investigador tiene sobre los procesos metodológicos, los sentimientos positivos o negativos que estos generan y la tendencia o intención conductual de aceptación o rechazo del proceso (p. 540).

Sabida la importancia que tienen la ciencia y la producción de conocimiento en las universidades, y que tal responsabilidad la ejerce fundamentalmente el cuerpo académico de estas instituciones, conviene conocer cómo se juzgan los propios docentes en cuanto a su capacidad para generar ciencia. Una actitud favorable propiciará un compromiso cada vez mayor en la generación y divulgación de conocimientos científicos eficaces en la atención de las necesidades sociales actuales. Una percepción desfavorable acerca de las propias capacidades, por el contrario, lo inhibirán. En efecto, tal y como afirma Vera (2012), "tanto una correcta valoración de la importancia, como el desarrollo de competencias científicas son centrales para que el docente pueda llevar a cabo de manera efectiva la función que se le exige dentro de la sociedad del conocimiento" (p. 10). Y los buenos investigadores no son otra cosa que personas educadas en la disciplina de la ciencia, que se ha convertido en investigadoras mediante la voluntad y el estudio (Aldana, Caraballo y Babativa, 2016).

Ahora bien, la competencia investigativa resulta de la conjunción de una serie de conocimientos, destrezas y actitudes complejas para el manejo de conceptos, técnicas, procedimientos y herramientas de manera efectiva en función de la productividad científica. Por eso el sistema educativo debe formar ciudadanos con competencias para investigar, pero, al mismo tiempo, esta es una de las áreas con mayores deficiencias. Por eso es necesario formar en competencias investigativas, estas son esenciales para adaptar, crear e innovar a partir de la articulación de las Tecnologías de la Información y la Comunicación (TIC) (Hernández, Prada y Ramirez, 2019).

Sobre este particular, conviene desglosar la competencia investigativa en sus múltiples facetas, que pueden amalgamarse en torno a tres ejes fundamentales:

1) La identificación y organización de la información tiene que ver con al arqueo, lectura, selección y sistematización de cualquier estudio previo o explicación teórica que pueda resultar útil tanto en el diseño de la investigación como en el posicionamiento teórico y el contraste de resultados con los de trabajos semejantes. Este eje incluye destrezas para la identificación de fuentes de información, la localización de bases de datos científicas y académicas, la distinción de publicaciones científicas y publicaciones de divulgación general, habilidad y criterio para seleccionar información en internet, la capacidad para seleccionar y ordenar los resultados de la búsqueda según el interés de la investigación, el conocimiento de los mecanismos de citación de autores, la capacidad de sintetizar conocimiento previo y la conciencia de la importancia de recurrir en la medida de lo posible a fuentes primarias de información.

2) En torno a la generación científica del conocimiento, se agrupan las capacidades para concentrar la atención en un aspecto problemático de la realidad, sopesar las posibilidades de emprender un estudio controlado de su fenomenología, diseñar un proyecto para el estudio del tema y llevarlo a término. Las habilidades, destrezas y actitudes necesarias para la generación de conocimiento nuevo tienen que ver con la capacidad de distinguir en la realidad circundante situaciones o problemas susceptibles de investigación, formular adecuadamente como problema de investigación una situación de la realidad, entender que toda investigación debe constituir un aporte original a un área de conocimiento, formular en términos de hipótesis o preguntas de investigación un problema científico, distinguir entre diversas metodologías la más adecuada a un problema determinado, capacidad de juicio para seleccionar adecuadamente los participantes de un estudio, seleccionar y diseñar instrumentos de recolección de datos, dominio de herramientas básicas de análisis estadístico para el 
tratamiento de los resultados, capacidad para reflejar resultados en tablas o gráficos, capacidad para contrastar resultados propios con los de otras investigaciones y conocimiento de los elementos éticos que deben seguirse cuando en una investigación participan seres humanos.

3) La divulgación de conocimientos involucra todas las posibilidades de hacer de dominio público los resultados de investigación, escoger los espacios más apropiados según la relevancia de los resultados y dar formato al reporte de investigación de acuerdo con los principios y acuerdos de la comunidad científica. En este sentido, un investigador competente debe conocer los distintos escenarios (reuniones científicas y publicaciones) usuales para la divulgación de la investigación, poder adecuar el formato de los resultados de la investigación al contexto en el que se presentan, participar en eventos en los que se divulgan los resultados de investigaciones, reconocer la importancia de la estandarización de normas para las publicaciones científicas, capacidad para componer y redactar un informe de investigación, participar en redes para la construcción colectiva de conocimiento, integrarse en comunidades virtuales para la construcción colectiva de conocimiento, participar en proyectos colaborativos a través del uso de TIC y capacidad para seleccionar apropiadamente el medio de divulgación de una investigación de acuerdo con la pertinencia y alcance de los resultados.

\section{Metodología}

El estudio que se reporta es de carácter cuantitativo, centrado en un análisis descriptivo correlacional-causal de los ítems de un test y utiliza los procedimientos y técnicas estadísticas clásicos para estudios psicométricos según lo ha definido la International Test Commission (2017). En consecuencia, en el presente estudio se efectúa un análisis de ítems bajo la teoría clásica y el análisis factorial en sus versiones exploratoria y confirmatoria.

\subsection{Muestra y selección de participantes}

Se trabajó con dos muestras. La primera estuvo constituida por 30 personas ( 18 hombres y 12 mujeres) entre 29 y 71 años. La edad promedio fue de 45.03 años (DT = 9.75 años). Los sujetos que la constituyeron fueron docentes de la UFPS, fueron contactados personalmente en su espacio de trabajo y participaron de forma voluntaria. Los profesores fueron seleccionados mediante método probabilístico sistemático, de acuerdo con su afiliación por departamento en las distintas facultades de la universidad. La justificación de la constitución de esta primera muestra fue calibrar la escala antes de su aplicación definitiva haciendo un estudio de sus propiedades métricas.

Una vez obtenida la versión definitiva y contando con el consentimiento informado de cada participante, se aplicó un cuestionario sobre datos sociodemográficos y se administró la escala definitiva. La segunda muestra estuvo integrada por 136 docentes de ambos sexos ( 110 hombres equivalente al $80.9 \%$, y 26 mujeres equivalente al $19.1 \%)$. El cálculo de la muestra tuvo un margen de error de $4.81 \%$ y un nivel de confianza de $95 \%$ en un universo de 725 docentes según registros de la Oficina de Recursos Humanos de la UFPS. La edad media de los participantes fue de 44.12 años (DT = 10.33 años). De nuevo, los docentes participaron de forma voluntaria y fueron localizados en sus espacios de trabajo. Para su selección y la aplicación del instrumento se siguió el mismo procedimiento que en la primera muestra.

\subsection{Instrumentos}

Cuestionario de las características de los participantes. Se elaboró un cuestionario ad hoc para obtener información sobre las características sociodemográficas de los sujetos (sexo, edad, título profesional, formación a nivel de postgrado, experiencia en investigación, participación en proyectos y publicaciones).

Perfil del docente universitario sobre productividad científica. El instrumento considera tres dimensiones: a) Manejo de información con 12 ítems codificados como InfoP $_{i}$ con $1 \leq \mathrm{i} \leq 12$; b) Generación de conocimiento 
con 14 ítems codificados como $\boldsymbol{G e n P}_{\boldsymbol{i}}$ con $1 \leq \mathrm{i} \leq 14$; c) Divulgación con 9 ítems codificados como $\operatorname{Divulg}_{i}$ con $1 \leq \mathrm{i} \leq 9$. Todos ellos se califican mediante una escala Likert con cinco niveles de respuesta.

\subsection{Procedimiento}

El protocolo de administración estuvo a cargo del grupo de investigadores. La aplicación de las pruebas fue de aproximadamente 10 minutos y se llevó a cabo en los espacios de trabajo de los docentes durante el segundo semestre del año 2019. Antes de la aplicación del instrumento los sujetos firmaron una carta de consentimiento mediante la cual hacían constar que fueron informados de los fines investigativos de la evaluación y la confidencialidad de los datos obtenidos.

\subsection{Análisis de datos}

Con los datos de la primera muestra se analizó la confiabilidad, validez y sensibilidad cultural de la versión inicial de la Escala de Productividad Científica - EPC. En el análisis de la versión inicial, el valor del Alfa de Cronbach de la escala global fue de 0.94. En la Tabla 1 se muestra la estructura de la escala en su versión inicial y dado que el valor de los índices de discriminación de los ítems osciló entre 0.40 y 0.77 , sugiere una adecuada capacidad de discriminación de los ítems. Por esta razón se mantuvo la cantidad de ítems originales en la versión traducida y se procedió a aplicar la escala a la muestra definitiva con el fin de evaluar su estructura factorial y realizar los análisis subsiguientes.

Tabla 1

Estadísticas de total de elemento

\begin{tabular}{|c|c|c|c|c|}
\hline Ítems & $\begin{array}{c}\text { Media de escala si el } \\
\text { elemento se ha suprimido }\end{array}$ & $\begin{array}{l}\text { Varianza de escala si el } \\
\text { elemento se ha suprimido }\end{array}$ & $\begin{array}{l}\text { Correlación total de } \\
\text { elementos corregida }\end{array}$ & $\begin{array}{c}\text { Alfa de Cronbach si el } \\
\text { elemento se ha suprimido }\end{array}$ \\
\hline InfoP1 & 142.03 & 282.447 & .679 & .952 \\
\hline InfoP2 & 142.30 & 278.769 & .640 & .952 \\
\hline InfoP3 & 142.03 & 280.102 & .687 & .952 \\
\hline InfoP4 & 142.43 & 279.840 & .576 & .952 \\
\hline InfoP5 & 141.97 & 285.826 & .687 & .952 \\
\hline InfoP6 & 142.30 & 280.079 & .693 & .951 \\
\hline InfoP7 & 142.20 & 283.614 & .527 & .953 \\
\hline InfoP8 & 142.03 & 279.206 & .765 & .951 \\
\hline InfoPg & 142.20 & 282.372 & .460 & .954 \\
\hline InfoP10 & 142.13 & 284.809 & .721 & .952 \\
\hline InfoP11 & 142.00 & 289.310 & .457 & .953 \\
\hline InfoP12 & 142.70 & 278.355 & .585 & .952 \\
\hline GenP1 & 142.23 & 281.151 & .705 & .951 \\
\hline GenP2 & 142.10 & 281.886 & .779 & .951 \\
\hline GenP3 & 141.80 & 287.062 & .627 & .952 \\
\hline GenP4 & 142.03 & 285.826 & .703 & .952 \\
\hline GenP5 & 142.00 & 285.172 & .657 & .952 \\
\hline GenP6 & 141.83 & 283.730 & .799 & .951 \\
\hline GenP7 & 142.23 & 283.978 & .753 & .951 \\
\hline GenP8 & 142.23 & 280.392 & .736 & .951 \\
\hline GenP9 & 141.90 & 282.162 & .730 & .951 \\
\hline GenP10 & 142.13 & 286.326 & .536 & .953 \\
\hline GenP11 & 141.93 & 290.961 & .454 & .953 \\
\hline GenP12 & 142.37 & 283.895 & .489 & .953 \\
\hline GenP13 & 142.10 & 285.128 & .689 & .952 \\
\hline
\end{tabular}




\begin{tabular}{lcccc}
\hline Ítems & $\begin{array}{c}\text { Media de escala si el } \\
\text { elemento se ha suprimido }\end{array}$ & $\begin{array}{c}\text { Varianza de escala si el } \\
\text { elemento se ha suprimido }\end{array}$ & $\begin{array}{c}\text { Correlación total de } \\
\text { elementos corregida }\end{array}$ & $\begin{array}{c}\text { Alfa de Cronbach si el } \\
\text { elemento se ha suprimido }\end{array}$ \\
\hline GenP14 & 141.83 & 285.385 & .711 & .952 \\
\hline DivulgP1 & 142.40 & 283.697 & .566 & .952 \\
\hline DivulgP2 & 142.23 & 281.840 & .676 & .952 \\
\hline DivulgP3 & 142.60 & 289.421 & .453 & .956 \\
\hline DivulgP4 & 142.03 & 283.620 & .679 & .952 \\
\hline DivulgP5 & 142.30 & 278.631 & .675 & .952 \\
\hline DivulgP6 & 142.77 & 275.909 & .676 & .952 \\
\hline DivulgP7 & 142.57 & 279.289 & .580 & .952 \\
\hline DivulgP8 & 142.93 & 283.582 & .404 & .954 \\
\hline DivulgP9 & 142.70 & 276.907 & .522 & .954 \\
\hline
\end{tabular}

Fuente. Elaboración propia

Un análisis exploratorio minucioso sugirió un especial tratamiento de casos atípicos denominados casos Heywood. En efecto, se identificó la presencia de seis casos irregulares que afectaban sustancialmente el comportamiento de la muestra, por lo que se procedió a su imputación para la conformación de una muestra definitiva de 130 docentes.

Con la muestra definitiva se realizaron dos análisis. En primer lugar, se evaluó la estructura factorial, para lo cual se realizó un Análisis Factorial Exploratorio (AFE) con rotación oblicua; ello, con el fin de analizar la estructura factorial del instrumento y la ubicación de los ítems en la misma. Posteriormente, se aplicó un Análisis Factorial Confirmatorio (AFC) a fin de comprobar la estructura factorial de la escala, su pertinencia teórica y la calidad del ajuste.

Dada la naturaleza ordinal de los datos y su alejado comportamiento al supuesto de normalidad multivariante, se optó por métodos alternativos de máxima verosimilitud. Los métodos basados en Mínimos Cuadrados Ordinarios (Ordinary Square Minimums - OSM) han mostrado mejores resultados con datos ordinales (Forero, Maydeu-Olivares \& Gallardo-Pujol, 2009; Lee, Zhang \& Edwards, 2012). Entre este conjunto de métodos destaca el análisis paralelo y su aplicación con Mínimos Cuadrados no Ponderados (Unweighted Square Minimums - ULS), que fue adoptado en este análisis, según la opinión de Lloret-Segura, Ferreres-Traver, Hernández-Baeza y TomásMarco (2014), por su adecuación a la naturaleza de las variables. Para su aplicación, se consideró como método de entrada de datos la matriz de correlaciones policóricas.

Durante la fase confirmatoria se seleccionó un software que dispusiera de los distintos métodos de estimación y formatos de entrada de datos con el fin de lograr mayor precisión en los resultados. El proframa FACTOR 7.0 (Lorenzo-Seva \& Ferrando, 2006) permite trabajar con la versión exploratoria y confirmatoria de forma simultánea y con distintos tipos de la matriz de entrada de los datos.

Para evaluar la adecuación del modelo se utilizaron, tal y como recomienda la literatura (Tanaka, 1993), diversos índices de ajuste. Para la interpretación se tuvieron en cuenta los valores sugeridos por Hu \& Bentler (1999): el estadístico chi-cuadrado relativo; el índice de ajuste comparativo (Comparative Fit Index - CFI) valores mayores a .90 e idealmente superiores de .95, indican un ajuste adecuado del modelo (Hu \& Bentler, 1998); el error cuadrático medio de aproximación (Root Mean Square Error Adjustment - RMSEA) que no debe exceder de 0.06; y residual estandarizado de la raíz cuadrada media (Residual of the Mean Square Root - RMSR), que se consideran apropiado cuando existen valores inferiores a 0.08 (Steiger, 2016). 


\section{Resultados y discusión}

\subsection{Evaluación de la dimensionalidad}

Se analizó la estructura de la escala con tres, cuatro, cinco y seis factores (ver Tabla 2). La solución inicial de tres factores no agrupó los ítems según la propuesta teórica original de tres factores que constituyen el modelo teórico inicial. Al adoptar un criterio libre para la rotación de los factores se encontró una estructura factorial de seis factores coherente para la solución. Esta solución, supera adecuadamente los supuestos de aplicación (test de Kaiser-Meyer-Olkin - KMO y esfericidad de Barlett) (López-Aguado y Gutiérrez-Provecho, 2019) y reportó un porcentaje elevado de varianza explicada que hizo viable su consideración. Las otras soluciones (cuatro y cinco factores), evidenciaron la existencia de factores infrarrepresentados (con un solo ítem), lo que dificultó su justificación teórica.

La solución de seis factores explicó en conjunto el $66.78 \%$ de la varianza del modelo. En el primer factor, que explica el $42.65 \%$, se agruparon diez ítems: ocho ítems de la dimensión de generación del conocimiento y dos ítems de manejo de información. El segundo factor explica el $7.44 \%$ de la varianza y está conformado por 5 ítems relacionado con competencias metodológicas. Los factores III y IV que explican el $5.42 \%$ y $4.02 \%$ respectivamente de la varianza están relacionados en su mayoría con la divulgación del conocimiento y un par de ítems de la cada una de las dos dimensiones restantes. Los factores $\mathrm{V}$ y $\mathrm{VI}$, explican cada uno el $3.72 \%$ y el 3.51\% y están relacionados principalmente con la identificación de las fuentes de la información y el manejo de la información derivada de la búsqueda. La Tabla 2 muestra la organización del conjunto de ítems por factores derivados en esta fase exploratoria.

Tabla 2

Matriz de estructura cargas factoriales y configuración factorial derivada del AFE

\begin{tabular}{ccccccc}
\hline & \multicolumn{7}{c}{ Componente } \\
\cline { 2 - 7 } G2 & $\mathbf{1}$ & $\mathbf{2}$ & $\mathbf{3}$ & $\mathbf{4}$ & $\mathbf{5}$ & $\mathbf{6}$ \\
\hline G6 & .848 & .339 & .506 & .501 & .419 & .195 \\
\hline G5 & .818 & .487 & .735 & .574 & .555 & .106 \\
\hline G4 & .798 & .301 & .647 & .476 & .495 & -.008 \\
\hline G1 & .790 & .333 & .572 & .559 & .452 & .012 \\
\hline G8 & .757 & .628 & .665 & .523 & .515 & .003 \\
\hline G3 & .741 & .256 & .462 & .486 & .461 & .145 \\
\hline I10 & .737 & .521 & .488 & .534 & .616 & .197 \\
\hline G7 & .727 & .446 & .662 & .581 & .495 & -.087 \\
\hline I12 & .567 & .350 & .197 & .071 & .379 & .439 \\
\hline D6 & .417 & .898 & .469 & .384 & .430 & .279 \\
\hline D8 & .359 & .886 & .450 & .361 & .331 & .224 \\
\hline D7 & .342 & .883 & .450 & .410 & .465 & .259 \\
\hline D9 & .488 & .771 & .644 & .422 & .477 & .103 \\
\hline D3 & .465 & .664 & .650 & .441 & .319 & .144 \\
\hline D4 & .453 & .362 & .811 & .478 & .474 & .273 \\
\hline D2 & .459 & .538 & .798 & .505 & .446 & .240 \\
\hline & & & & & & \\
\hline
\end{tabular}




\begin{tabular}{|c|c|c|c|c|c|c|}
\hline & \multicolumn{6}{|c|}{ Componente } \\
\hline & 1 & 2 & 3 & 4 & 5 & 6 \\
\hline $\mathrm{D} 1$ & .509 & .603 & .777 & .436 & .422 & .187 \\
\hline D5 & .618 & .599 & .763 & .567 & .502 & .170 \\
\hline 13 & .411 & .491 & .694 & .286 & .489 & .408 \\
\hline G14 & .571 & .249 & .588 & .560 & .498 & -.091 \\
\hline G10 & .529 & .397 & .425 & .864 & .385 & .114 \\
\hline G12 & .452 & .516 & .537 & .834 & .370 & .072 \\
\hline G9 & .700 & .356 & .532 & .791 & .552 & .144 \\
\hline G13 & .623 & .416 & .678 & .780 & .439 & -.001 \\
\hline G11 & .502 & .238 & .530 & .701 & .369 & .229 \\
\hline 17 & .439 & .400 & .411 & .370 & .871 & .328 \\
\hline 18 & .446 & .551 & .479 & .496 & .792 & .249 \\
\hline 111 & .598 & .327 & .450 & .396 & .765 & .134 \\
\hline 19 & .347 & .259 & .361 & .202 & .701 & .170 \\
\hline 16 & .435 & .472 & .339 & .594 & .644 & .326 \\
\hline 15 & .432 & .067 & .180 & .298 & .506 & .393 \\
\hline I1 & .206 & .290 & .332 & .280 & .257 & .730 \\
\hline 12 & .363 & .440 & .391 & .316 & .433 & .684 \\
\hline 14 & .428 & .421 & .410 & .260 & .515 & .648 \\
\hline
\end{tabular}

Método de extracción: análisis de componentes principales.

Método de rotación: Promax con normalización Kaiser.

Fuente. Elaboración propia

\subsection{Análisis Factorial Confirmatorio}

La estructura de tres factores fue sometida a un Análisis Factorial Confirmatorio (AFC). Una inspección del análisis descriptivo univariado indica que la distribución de los ítems de naturaleza ordinal es asimétrica y tiene valores altos de curtosis, por lo que se sugiere el uso de la matriz policórica (Muthén \& Kaplan, 1985). Los valores de las correlaciones policóricas oscilan entre 0.22 y 0.69 . El método de extracción de factores asumido fue el Análisis Paralelo (Timmerman \& Lorenzo-Seva, 2011) y el método de estimación de parámetros fue el Mínimos Cuadrado No Ponderado (ULS -Unweighted Least Squares) (Jöreskog, 1977). Así, la solución alcanza su mínimo y el modelo está identificado.

Al evaluar el ajuste del modelo, se observa que se obtuvo un conjunto de índices de ajuste satisfactorios (ver Tabla 3). Los principales fueron los siguientes: Chi-cuadrado relativo $\left(\mathrm{X}^{2} / \mathrm{gl}\right)=1.61$; Non-Normed Fit Index $-\mathrm{NNFI=}$ 0.984; CFI= 0.988; Adjusted Goodness of Fit Index - AGFI=0.991, todos superiores a 0.95, lo cual representa un óptimo ajuste de los datos al modelo teórico. Un índice importante es el RMSEA=0.048. Puesto que se encuentra por debajo de 0.05 ratifica la tesis del excelente ajuste de la escala. El RMSR del modelo fue 0.0252, con un intervalo de confianza que oscila entre 0.022 y 0.027 . Puesto que contiene el valor medio esperado del RMSR (0.0252) se considera un modelo aceptable. 
Tabla 3

Índices de ajuste

\begin{tabular}{lll}
\hline Índices de ajuste & Valor & IC Bootstrap 95\% \\
\hline Root Mean Square Error of Approximation (RMSEA) & 0.048 & $0.0414-0.0546$ \\
Residual of the Mean Square Root (RMSR) & 0.0252 & $0.022-0.027$ \\
Fit Function Chi Square with 35 degrees of freedom & $56.551(\mathrm{p}=0.012028)$ & \\
Chi cuadrado relativo Chi Square/df (56.551/35) & $1.61(<3)$ & \\
Non-Normed Fit Index (NNFI; Tucker \& Lewis) & 0.984 & $0.979-0.993$ \\
Comparative Fit Index (CFI) & 0.988 & $0.984-0.994$ \\
Schwarz's Bayesian Information Criterion (BIC) & 229.413 & $195.822-243.247$ \\
Goodness of Fit Index (GFI) & 0.993 & $0.992-0.996$ \\
Adjusted Goodness of Fit Index (AGFI) & 0.991 & $0.989-0.994$ \\
Goodness of Fit Index without diagonal values (GFI) & 0.99 & $0.987-0.994$ \\
Goodness of Fit Index without diagonal values(AGFI) & 0.987 & $0.983-0.992$ \\
\hline
\end{tabular}

Fuente. Elaboración propia

El modelo final ajustado se muestra en la Figura 1. Se confirma en ella la estructura de seis factores en un AFC de segundo orden. Se mantiene la estructura original de tres factores (generación, divulgación y manejo de la información) en el primer orden y se amplía a un modelo de segundo orden en el que cada variable latente se subdivide en dos nuevas, ampliando así la comprensión de la productividad científica a partir de conceptos relacionados y con nivel conceptual diferenciado.

Figura 2

Modelo final ajustado

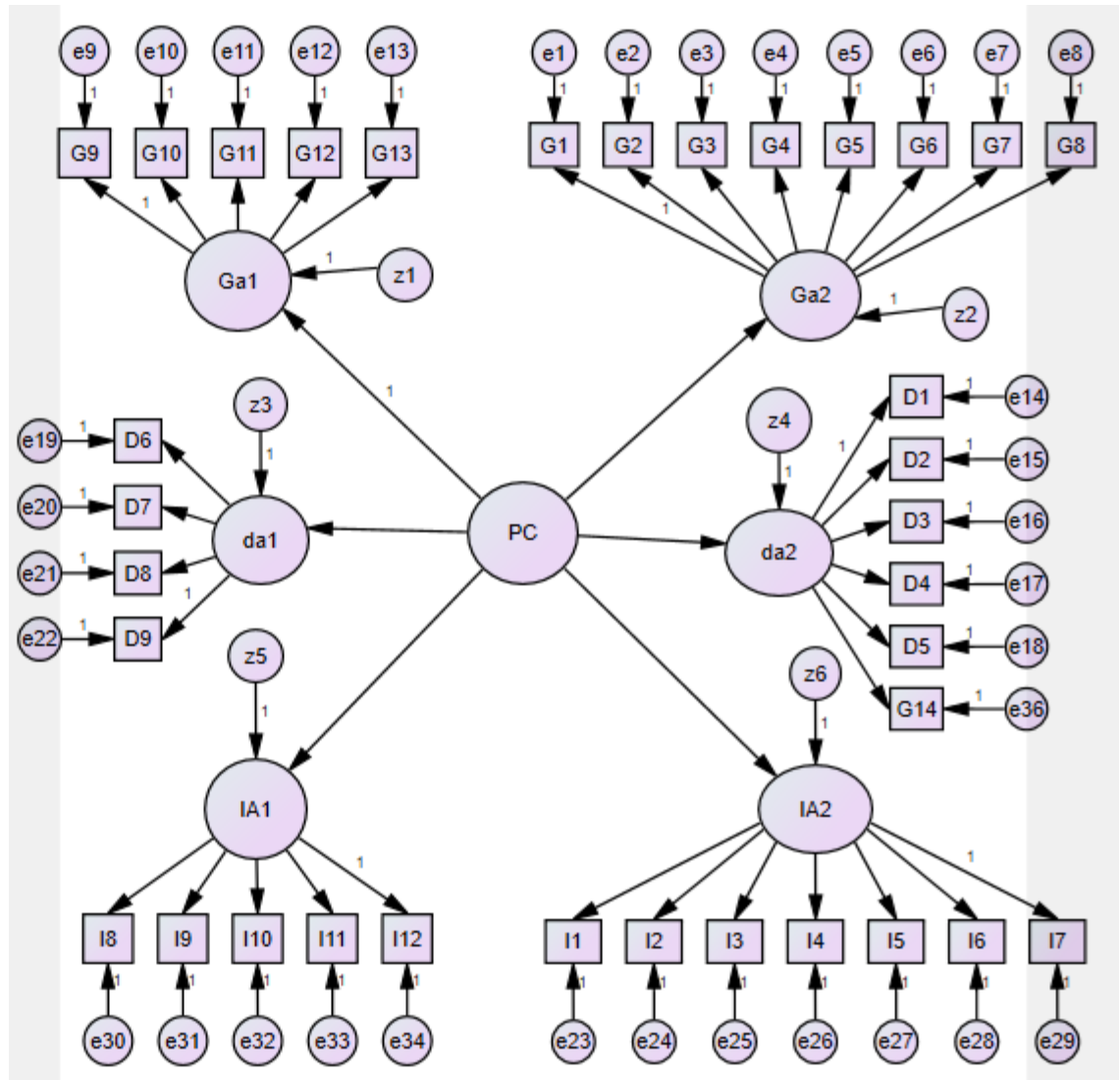


El AFC, en consecuencia, más que confirmar la estructura original, derivó en una ampliación de seis factores jerarquizados en torno a la productividad científica. Tales factores son los siguientes: habilidades analíticas, habilidades metodológicas, distinción de entornos, comunicación de resultados y búsqueda y manejo de información. Todos ellos se explican a partir de un conjunto de indicadores claramente relacionados y justificados teóricamente. Una visión detallada de la estructura de cada factor se muestra en la Tabla 4.

Tabla 4

Relación de ítems de acuerdo a los resultados del AFC y el modelo ajustado

\begin{tabular}{|c|c|c|c|}
\hline Nivel I & Nivel II & Item & Indicador \\
\hline \multirow{13}{*}{$\begin{array}{l}\text { Generación del } \\
\text { conocimiento }\end{array}$} & \multirow{5}{*}{$\begin{array}{l}\text { Habilidades } \\
\text { analíticas }\end{array}$} & G9 & Selección de instrumentos adecuados \\
\hline & & G10 & Manejo de herramientas de análisis estadístico \\
\hline & & G11 & Elaboración de tablas o gráficos \\
\hline & & $\mathrm{G} 12$ & Reconocimiento de métodos para analizar datos \\
\hline & & G13 & Contraste de resultados obtenidos \\
\hline & \multirow{8}{*}{$\begin{array}{l}\text { Habilidades } \\
\text { metodológicas }\end{array}$} & G1 & Identificación de problemas de investigación \\
\hline & & G2 & Formulación de problemas de investigación \\
\hline & & G3 & Capacidad para justificar la investigación \\
\hline & & G4 & Formulación de hipótesis de investigación \\
\hline & & G5 & Fundamentación de problemas de investigación \\
\hline & & G6 & Definición de objetivos y propósitos \\
\hline & & G7 & Diseños metodológicos adecuados \\
\hline & & G8 & Definición y cálculo de muestras \\
\hline \multirow{10}{*}{$\begin{array}{l}\text { Divulgación del } \\
\text { conocimiento }\end{array}$} & \multirow{4}{*}{$\begin{array}{l}\text { Distinción } \\
\text { entornos }\end{array}$} & D6 & Participación en redes de conocimiento \\
\hline & & D7 & Participación en comunidades virtuales \\
\hline & & $\mathrm{D} 8$ & Participación en proyectos colaborativos \\
\hline & & D9 & Selección de medios de divulgación \\
\hline & \multirow{6}{*}{$\begin{array}{l}\text { Comunicación } \\
\text { de los } \\
\text { resultados }\end{array}$} & D1 & Identificación de espacios para la divulgación \\
\hline & & $\mathrm{D} 2$ & Adaptación de los resultados a los formatos de presentación \\
\hline & & D3 & Participación en eventos científicos \\
\hline & & D4 & Reconocimiento de normas de presentación de manuscritos \\
\hline & & D5 & Redacción de informes de investigación \\
\hline & & G14 & Conocimiento de lineamientos éticos \\
\hline \multirow{12}{*}{$\begin{array}{l}\text { Localización de } \\
\text { fuentes } \\
\text { informativas }\end{array}$} & \multirow{5}{*}{$\begin{array}{l}\text { Búsqueda de la } \\
\text { información }\end{array}$} & 18 & Selección de fuentes de información \\
\hline & & 19 & Argumentación de las ideas \\
\hline & & 110 & Síntesis ordenada de los resultados de la búsqueda \\
\hline & & $\mathrm{I11}$ & Fundamentación en otros autores \\
\hline & & 112 & Uso de fuentes primarias \\
\hline & \multirow{7}{*}{$\begin{array}{l}\text { Manejo de la } \\
\text { información }\end{array}$} & 11 & Identificación de fuentes de información \\
\hline & & 12 & Identificación de bases de datos científicas \\
\hline & & 13 & Identificación de publicaciones científicas \\
\hline & & 14 & Ubicación de información disponible en internet \\
\hline & & 15 & Uso de información disponible en internet \\
\hline & & 16 & Uso de plataformas especializadas \\
\hline & & 17 & Organización de los resultados de la búsqueda \\
\hline
\end{tabular}

Fuente. Elaboración propia 


\section{Discusión}

El modelo planteado prioriza las competencias relacionadas con la generación del conocimiento científico en relación con la divulgación y la localización de la información. En primera instancia sobresalen las competencias relacionadas con la habilidad analítica, es decir con las competencias y conocimientos para analizar la información. Esta actividad se ubica por encima de las habilidades metodológicas, esto es, las relacionadas con la identificación y formulación de problemas de investigación y con la capacidad para discernir entre métodos de investigación con la finalidad de realizar una selección acorde con la naturaleza de los datos y proponer un diseño metodológico para responder a los propósitos de la investigación (Gómez-Delgado y Villalobos-Galvis, 2014).

En este modelo, las competencias relacionadas con el manejo de la información, es decir, su búsqueda y utilización, aparecen en tercera posición. Resulta así que, si bien se valora su importancia, quedan relegadas en relación con la generación del conocimiento y su divulgación. Estos resultados se corresponden con el perfil actual del docente universitario, que considera las competencias relativas a la administración de la información y el empleo de tecnologías como características inherentes a su formación y desempeño como profesional universitario, pero que adolece aún de una adecuada formación metodológica. El despliegue de habilidades tecnológicas y de localización de información en entornos digitales se relaciona con la consolidación de una generación de nativos digitales, que otorgan mayor preponderancia a las capacidades analíticas y al empleo de metodologías, pues reconocen su impericia en este ámbito (Hernández, Prada y Ramirez, 2019) . Ello, a pesar de que las habilidades para el uso de metodologías constituyen uno de los rasgos indispensables para el desempeño de las labores propias del profesor universitario, principalmente en su rol como investigador.

Una evidencia de esta falta de destreza en el conocimiento y aplicación de los métodos para generar nuevo conocimiento tiene que ver con el hecho de que, en los proyectos emprendidos desde las universidades, una partida significativa de los presupuestos se destina al asesoramiento en materia de análisis de la información con el fin de dar respuestas a hipótesis e interrogantes del estudio (Burdiles, Castro y Simian, 2019). Sin embargo, en lo que concierne a sus habilidades para el manejo de información los docentes no reportan déficit.

La divulgación del conocimiento científico es una actividad que cobra cada vez mayor relevancia en las universidades latinoamericanas. A este respecto, en el modelo se despliegan dos dimensiones interesantes. La primera está relacionada con la distinción de entornos para la difusión de los resultados de la investigación en eventos caracterizados por su afinidad académica y su vinculación con la producción científica del conocimiento (Ledón y Agramonte, 2005). La participación en estos eventos se hace de forma personal o a través de espacios virtuales, escenarios que los docentes reconocen con facilidad (Crespo, 2015), aunque existe una baja puntuación en los entornos online (Vega et al, 2019).

Por otra parte, se encuentran las habilidades para comunicar los resultados, que consiste en un conjunto de destrezas relacionadas con la conformación del informe de la investigación según los estándares de la comunidad científica para la divulgación de la investigación (Arzola, 2019). En el otro extremo, destacan las competencias relacionadas con la presentación de los resultados. En este sentido, resulta prioritario dar rigor científico y ajustar al formato académico que la comunidad exige de los manuscritos propuestos para su publicación. Es en este punto donde la competencia relacionada con la comunicación escrita cobra mayor valor e invita a los docentes a desempeñarse según los estándares internacionales en la presentación y publicación de conocimiento generado, además de considerar los aspectos éticos necesarios (Ramírez, Martínez y Castellanos, 2012), principalmente si los sujetos de investigación son personas ¿en situación de vulnerabilidad? o niños (Castro y García, 2018). 


\section{Conclusiones}

Como conclusión, el modelo ajustado mantiene la estructura original de tres factores (generación, divulgación y manejo de la información) en el primer orden y se amplía a un modelo de segundo orden en el que cada variable latente se subdivide en dos nuevas, ampliando así la comprensión de la productividad científica a partir de conceptos relacionados y con nivel conceptual diferenciado.

Asímismo, el Análisis Factorial Confirmatorio más que confirmar la estructura original, derivó en una ampliación de seis factores jerarquizados en torno a la productividad científica: habilidades analíticas, habilidades metodológicas, distinción de entornos, comunicación de resultados y búsqueda y manejo de información; explicados a partir de un conjunto de indicadores relacionados y justificados teóricamente.

Finalmente, el modelo generado resulta suficientemente explicativo de las distintas variables relacionadas con la producción científica del conocimiento por parte de docentes universitarios, amplio en sus alcances y parco en su diseño. El modelo, por lo demás, invita a la réplica y constituye un punto de partida para modelos complejos que profundicen la perspectiva del ejercicio de la investigación en los espacios de la educación superior.

\section{Referencias bibliográficas}

Aldana, G., Caraballo, G. y Babativa, D. (2016). Escala para medir actitudes hacia la investigación (eacin): validación de contenido y confiabilidad. Aletheia, 8(2), 104-121. http://dx.doi.org/10.11600/21450366.8.2aletheia.104.121

Arzola, D. (coord.) (2019). Procesos formativos en la investigación educativa. Diálogos, reflexiones, convergencias y divergencias. Chihuahua, México: Red de Investigadores Educativos Chihuahua AC.

Beraza, J. y Rodríguez, A. (2007). La evolución de la misión de la universidad. Revista de Dirección y Administración de Empresas, 14, 25-56. https://www.ehu.eus/ojs/index.php/rdae/article/download/11424/10524

Blanco, N. (2001). Una técnica para la medición de actitudes sociales. Revista de ciencias sociales, 7(1), 45-54. http://dx.doi.org/10.31876/rcs.v7i1.25120

Blanco, N. y Alvarado, M. (2005). Escala de actitud hacia el proceso de investigación científico social. Revista de ciencias sociales, 11(3), 537-544. http://dx.doi.org/10.31876/rcs.v11i3.25304

Bogado de Scheid, L. y Fedoruk, S. (2011). Rol de las universidades. Docencia - investigación y Extensión: Una relación imprescindible. En XI Congreso Iberoamericano de Extensión Universitaria Integración extensión, docencia e investigación para la inclusión y cohesión social. Santa Fe, Argentina: Universidad Nacional del Litoral. http://www.unl.edu.ar/iberoextension/dvd/archivos/ponencias/mesa3/rol-de-las-universidadesdoc.pdf

Burdiles, P., Castro, M. y Simian, D. (2019). Planificación y factibilidad de un proyecto de investigación clínica. Revista medica clinica las condes, 30(1), 8-18. https://doi.org/10.1016/j.rmclc.2018.12.004

Canto, J. (1994). Psicología social e influencia. Estrategias de poder y procesos de cambio. Málaga: Aljibe.

Castro, N. y García, S. (2018). Principios éticos rectores de las investigaciones de tercer nivel o grado académico en Ciencias Médicas. Revista Cubana de Educación Médica Superior, 32(2), 1-12.

https://www.medigraphic.com/pdfs/educacion/cem-2018/cem182z.pdf 
Crespo, J. (2015). Eventos académicos virtuales: ideas alrededor de un estado de la cuestión. ASRI: Arte y sociedad. Revista de investigación, (8), 7. https://dialnet.unirioja.es/descarga/articulo/5029081.pdf

Forero, C., Maydeu-Olivares, A. \& Gallardo-Pujol, D. (2009). Factor analysis with ordinal indicators: A monte Carlo study comparing DWLS and ULS estimation. Structural Equation Modeling, 16(4), 625-641. https://doi.org/10.1080/10705510903203573

Gamboa, A., Hernández, C. y Prada, R. (2020). Competencias científicas, investigativas y comunicativas: experiencias desde una línea de investigación en enseñanza de las Ciencia. Plumilla Educativa, 25 (1), 1536. http://dx.doi.org/10.30554/pe.1.3827.2020

Gómez-Delgado, Y.A. \& Villalobos-Galvis, F.H. (2014). Competencias para la formulación de un proyecto de Investigación. Guía metodológica del proyecto INVESTIC para docentes investigadores. San Juan de Pasto: Editorial Universidad de Nariño

Hernández, C., Prada, R. y Ramirez, P. (2019). Competencias TIC e investigativas entre docentes de educación básica. Bogotá: Ecoe Ediciones -Universidad Francisco de Paula Santander.

Hu, L.-T., \& Bentler, P. (1998). Fit indices in covariance structure modeling: Sensitivity to underparameterized model misspecification. Psychological Methods, 3(4), 424-453. https://doi.org/10.1037/1082-989X.3.4.424

Hu, L.-T., \& Bentler, P. (1999). Cutoff criteria for fit indexes in covariance structure analysis: Conventional criteria versus new alternatives. Structural Equation Modeling, 6, 1-55.

https://doi.org/10.1080/10705519909540118

International Test Commission. (2017). The ITC Guidelines for Translating and Adapting Tests. https://www.intestcom.org/files/guideline_test_adaptation_2ed.pdf

Jöreskog, K. G. (1977). Factor analysis by least-squares and maximum-likelihood methods. En K. Enslein, A. Ralston, \& H. S. Wilf (Eds.), Statistical methods for digital computers (pp. 125-153). New York: Wiley.

Lee, C.-T., Zhang, G., \& Edwards, M. (2012). Ordinary least squares estimation of parameters in exploratory factor analysis with ordinal data. Multivariate Behavioral Research, 47, 314-339. https://doi.org/10.1080/00273171.2012.658340

Ledón, L. y Agramonte, A. (2005). Difusión de resultados de investigación: reflexiones desde el ejercicio de una ciencia ética y responsable. Revista Humanidades Médicas, 5(3). http://scielo.sld.cu/scielo.php?script=sci_arttext\&pid=S1727-81202005000300003\&lng=es

Lloret-Segura, S., Ferreres-Traver, A., Hernández-Baeza, A., \& Tomás-Marco, I. (2014). Exploratory Item Factor Analysis: A practical guide revised and up-dated. Anales De Psicología, 30(3), 1151-1169. https://doi.org/10.6018/analesps.30.3.199361

López-Aguado, M., y Gutiérrez-Provecho, L. (2019). Cómo realizar e interpretar un análisis factorial exploratorio utilizando SPSS. REIRE Revista d'Innovació i Recerca en Educació, 12(2), 1-14. http://doi.org/10.1344/reire2019.12.227057

Lorenzo-Seva, U., \& Ferrando, P. (2006). FACTOR: A computer program to fit the exploratory factor analysis model. Behavior Research Methods, 38, 88-91 (2006). https://doi.org/10.3758/BF03192753

Mas, Ó. (2011) El profesor universitario: sus competencias y formación. Profesorado, 15 (3), 195-211. http://www.ugr.es/local/recfpro/rev153COL1.pdf 
Ministerio de Educación Nacional. (16 de Septiembre de 2005). ¿Qué tanto promueven la investigación las universidades en Colombia? Centro Virtual de Noticias, 66-70.

http://www.mineducacion.gov.co/cvn/1665/fo-article-88439.pdf

Muthén, B., \& Kaplan, D. (1985). A Comparison of Some Methodologies for the Factor Analysis of Non-Normal Likert Variables. British Journal of Mathematical and Statistical Psychology, 38, 171-189. http://dx.doi.org/10.1111/j.2044-8317.1985.tb00832.x

Organisation for Economic Co-operation and Develoment (2007). Higher education and regions. Global competitive, locally engaged. Paris: OECD.

Orler, J. (2012) Docencia-Investigación: ¿una relación antagónica, inexistente o necesaria? Academia. Revista sobre enseñanza del Derecho, 19, 289-301.

http://www.derecho.uba.ar/publicaciones/rev_academia/revistas/19/docencia-investigacion-unarelacion-antagonica-inexistente-o-necesaria.pdf

Palacio, I. (2010). La investigación a través de los tiempos. Bogotá: Editorial Universidad del Rosario.

Plastino, Á. (2008). La universidad: lugar de investigación científica y transferencia tecnológica. Revista Educación Superior y Sociedad, 13(1), 63-72.

http://www.iesalc.unesco.org/ess/index.php/ess3/issue/view/36

Ramírez, D., Martínez, L. y Castellanos, O. (2012). Divulgación y difusión del conocimiento : las revistas científicas Bogotá : Universidad Nacional de Colombia.

Rodríguez, L. (2007). La teoría de la acción razonada. Implicaciones para el estudio de las actitudes. Investigación educativa duranguense, (7), 66-77. http://www.upd.edu.mx/PDF/Revistas/InvestigacionEducativaDuranguense7.pdf

Ruiz, J. (2010). Importancia de la investigación (Editorial). Revista Científica, 20(2), 125-126. https://produccioncientificaluz.org/index.php/cientifica/article/download/15542/15516

Sabater, J. (1989) Sobre el concepto de actitud. Anales de pedagogía, (7), 159-187. https://revistas.um.es/analespedagogia/article/view/287671

Sánchez, B. (2007). Integración docencia e investigación: un modelo didáctico para la producción y extensión del conocimiento en educación superior. En VII Reunión Nacional de Currículo y I Congreso Internacional de Calidad e Innovación en Educación Superior: Caracas: Universidad Simón Bolívar. http://www.cies2007.eventos.usb.ve/ponencias/152.pdf

Sancho Gil, J.M. 2001. Docencia e investigación en la universidad: una profesión, dos mundos. Educar, 28, 4160. https://www.raco.cat/index.php/Educar/article/view/20742

Sigal, V. (2009). La universidad y la investigación. Gestión universitaria, 1 (2). http://www.gestuniv.com.ar/gu_02/v1n2a2.htm

Steiger, J. (2016). Notes on the Steiger-Lind (1980) handout. Structural Equation Modeling, 23(6), 777-781. https://doi.org/10.1080/10705511.2016.1217487

Summers, G. (Ed.) (1984). Medición de actitudes. México: Trillas.

Tanaka, J. (1993). Multifaceted conceptions of fit in structural equation models. En K. A. Bollen \& J. S. Long (Eds.), Testing structural equation models (pp. 10-39). Newbury Park, CA: Sage 
Timmerman, M. \& Lorenzo-Seva, U. (2011). Dimensionality assessment of ordered polytomous items with parallel analysis. Psychological Methods, 16(2), 209-220. http://dx.doi.org/10.1037/a0023353

Vega, O., Rodriguez-Baena, L., Perez-Agustin, M., \& Melo-Solarte, D. S. (2019). Professors digital situation measurement: the case of a Colombian private university. Revista ESPACIOS, 40(36), 28. http://www.revistaespacios.com/a19v40n36/19403628.html

Vera, J. (2012). Competencias científicas de docentes de Educación Superior Tecnológica en México, Universidades, (52), 4-17. http://www.redalyc.org/articulo.oa?id=37326902002

Vergara, M., Gamboa, A. y Hernández, C. (2018). Políticas de investigación en educación superior: imaginarios instituidos de una universidad pública en Norte de Santander, Colombia. Revista Espacios, 39(43), 35. http://www.revistaespacios.com/a18v39n43/a18v39n43p35.pdf

Yoguez, A. (2009). ¿Cómo se evalúan las Universidades de Clase Mundial? Revista de la educación superior, 38(150), 113-120. http://publicaciones.anuies.mx/pdfs/revista/Revista150_S4A3ES.pdf

Esta obra está bajo una Licencia Creative Commons Attribución-NoCommercial 4.0 International

(cc) BY-NC 